\title{
Stretching Polymers in Droplet-Pinch-Off Experiments
}

\author{
F. Ingremeau and H. Kellay \\ Université Bordeaux 1, Laboratoire Ondes et Matière d'Aquitaine, (UMR 5798 CNRS), \\ 351 cours de la Libération, 33405 Talence France
}

(Received 6 July 2012; revised manuscript received 10 June 2013; published 14 October 2013)

\begin{abstract}
Droplet pinch off, which occurs when a drop of liquid detaches from a capillary, can be strongly modified in the presence of polymers, giving rise to long and slender filaments that thin slowly in time. However, little is known experimentally about the molecular conformations of the polymers in the filament itself. Since the thinning dynamics of these filaments can be used to extract macroscopic quantities of interest such as the elongational viscosity of polymer solutions, which is of importance in a variety of physical processes (electrospinning, spraying, or drag reduction), the link with the molecular scale dynamics would be an important step towards understanding the extensional properties of such solutions. We show here, experimentally, that the polymers are highly extended within this filament region and that the distribution of these extensions is stationary in time. We then determine the elongational viscosity from the full filament dynamics. Such a determination turns out to be consistent with the polymer extensions observed and also consistent with simple models of polymer elongational viscosities. The direct observation of stretched macromolecules within such suspended liquid filaments suggests that these filaments may be useful for preparing polymeric materials with specific anisotropy or for optical screening of biopolymers such as DNA.
\end{abstract}

DOI: 10.1103/PhysRevX.3.041002

Subject Areas: Fluid Dynamics, Soft Matter

\section{INTRODUCTION}

Complex fluids exhibit a variety of properties that set them apart from simple Newtonian fluids like water. A crucial difference between these two types of fluids is the presence of mesoscopic constituents, such as polymers or surfactant aggregates, which render the interaction of the complex fluid with an applied stress complicated because of the multiplicity of length and time scales that may enter into play. Understanding such fluids relies on understanding the complicated interactions between their constituents as well as their interactions with an external perturbation such as a flow field. For example, in both simple shear flows and elongational flows, polymer molecules may show a variety of conformations and nontrivial dynamics [1-4]. The coupling between the molecular configurations and the flow field is responsible for dramatic macroscopic manifestations such as elastic turbulence [5], inhibition of singularity formation [6], and drag reduction [7].

A powerful way to identify the differences in behavior between complex and Newtonian fluids is the spontaneous thinning and ensuing rupture of a column of liquid. The rupture of Newtonian liquid columns can be modeled using self-similar solutions for the neck profile and power laws for the thinning of the neck [8]. Some complex fluids, however, exhibit an inhibition of such singular dynamics, which is replaced, in a sudden fashion, by single time

Published by the American Physical Society under the terms of the Creative Commons Attribution 3.0 License. Further distribution of this work must maintain attribution to the author(s) and the published article's title, journal citation, and DOI. dynamics represented by exponential thinning of the neck [6,9-12]. Different classes of complex fluids have shown such a phenomenology: polymer solutions, corn starch solutions [13], and micellar phases of surfactant [14]. Such exponential thinning has become a powerful tool to characterize complex fluids, and especially polymer solutions and their elongational viscosity $[9,11,15,16]$, since the early work of Bazilevsky, Entov, and Rozhkov, and the later work of Entov and Hinch [17-19]. Despite its generality, the link between this macroscopic thinning behavior, dubbed visco-elasto-capillary thinning [19], and the microscopic properties of the solutions (such as the distribution of molecular extensions or orientations) remains an experimental challenge.

Here, we use solutions of long-chain polymers and examine the link between the microscopic properties of the solution and the thinning dynamics of a column of such liquid. In particular, we measure the stretching of the polymers within this flow as well as the resistance of the fluid to extension. Our results show that the polymer molecules are stretched by the flow in the visco-elastocapillary regime, with a distribution of lengths that remains stationary in time. We then show that the elongational viscosity of such solutions, obtained from measurements of the velocity field within the thinning neck as well as the shape of this neck, is large compared to their shear viscosity and has little growth in time, in accordance with the molecular extensions measured. The behavior of such filaments presents an ideal situation to explore the link between macroscopic and microscopic properties of complex fluids in extensional flows since both properties can be measured simultaneously: The macroscopic properties 
can be monitored through the shape of the neck and a measurement of the velocity field, while the microscopic properties can be monitored through molecular extension measurements. Furthermore, suspended liquid columns with stretched macromolecules embedded within them may prove very useful to prepare materials with specific anisotropies or for optical screening purposes of DNA molecules, for example.

\section{RESULTS}

First, let us describe the formation and dynamics of the filaments obtained by droplet detachment of polymer solutions from capillaries (see the Appendix for more details about the solution and the setup). Images of the drop breakup obtained in the microfluidic channel are shown in Fig. 1. After a first phase, where the neck between the detaching drop and the capillary is symmetrical with a fast thinning dynamics, a cylindrical filament forms between the drop and the capillary. The breakup dynamics of the fluid neck is followed by measuring the minimum neck diameter $h_{\min }$ versus time, as shown in Fig. 1. The breakup dynamics displays typical behavior for a polymeric fluid, whether for drops falling under gravity or in microfluidic channels [6,20-22]. The initial thinning is similar to that of a Newtonian fluid; the thinning dynamics of a similar viscosity Newtonian fluid is also plotted in the inset. The role of the polymer is therefore absent for these initial instants. When the thinning rate becomes strong enough, a second regime emerges where the thinning slows down drastically and is well approximated by an exponential decay of the form $h_{\min } \propto \exp \left(-\frac{1}{2} \dot{\varepsilon}_{0} t\right)$ with $\dot{\varepsilon}_{0}=10.5 \pm$ $0.5 \mathrm{~s}^{-1}$. This is the visco-elasto-capillary regime. For such a cylindrical filament, the flow is purely extensional, with a rate of elongation $\dot{\varepsilon}=\frac{\partial v_{z}}{\partial z}=-\frac{2}{h} \frac{\partial h}{\partial t}=\dot{\varepsilon}_{0}$ ( $z$ is parallel to the filament axis). The strength of the elongation rate in the neck region is crucial for polymer stretching within this region. We have therefore determined the elongation rate within the neck region from a measurement of the velocity field. Measurements of the local elongation rate from the variation of the velocity along the center line of the neck are shown in Fig. 1. This rate starts out small and goes through a sharp maximum near the transition to filament formation, reaching a value near $600 \mathrm{~s}^{-1}$, before decreasing and eventually saturating at a constant value near $\dot{\varepsilon}_{0}$ in the filament regime. Near the transition from the Newtonian behavior to the visco-elasto-capillary regime, the extension rate reaches higher values than the inverse relaxation time of the polymer solution, favoring the extension of the molecules. Besides the extension rate itself, a quantity of interest is the total strain $\varepsilon=\int \dot{\varepsilon} d t$, which is shown in the inset of Fig. 1. The instant $t=0$ is taken as

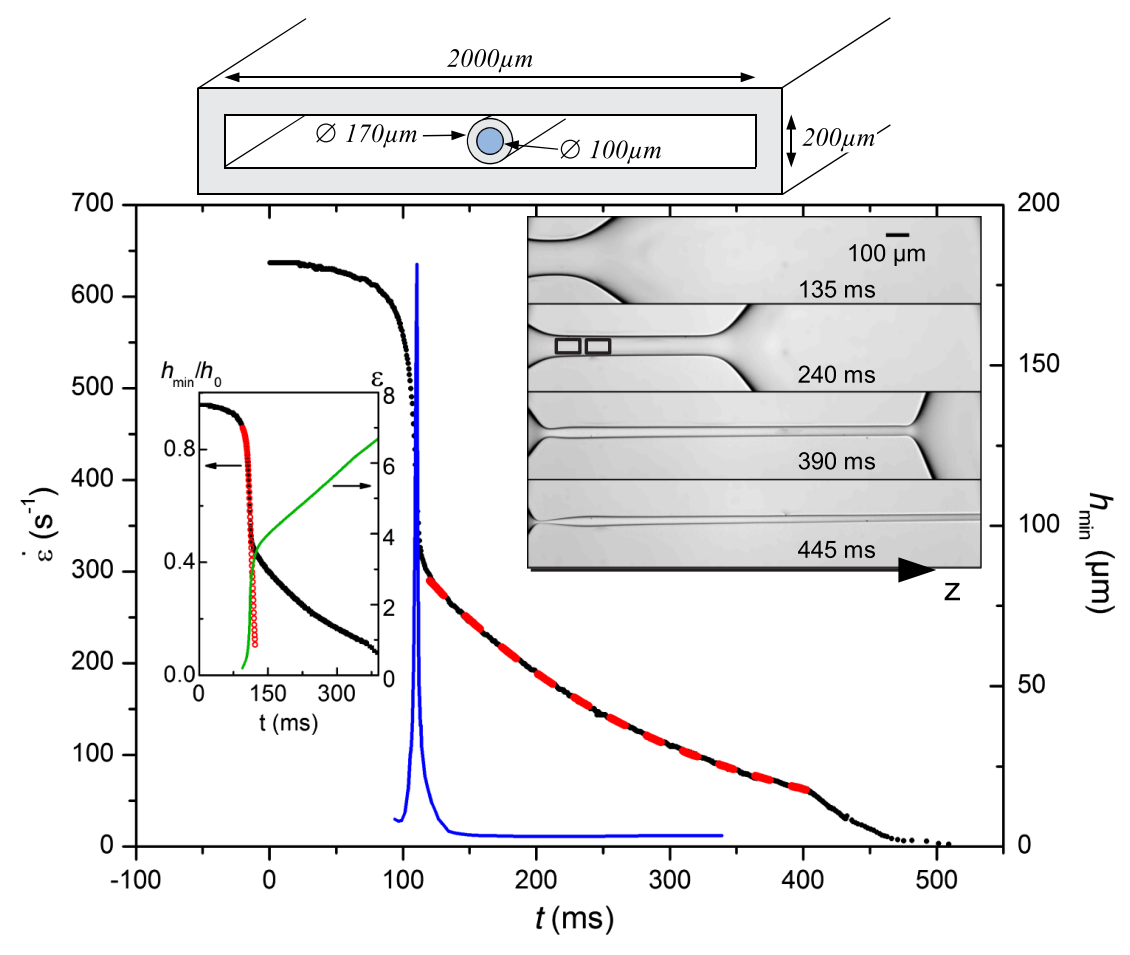

FIG. 1. Setup and thinning dynamics: Sketch of the channel used, which consists of a round capillary, to inject the polymer solution, embedded in a larger rectangular capillary for the coflowing liquid. We show images of the thinning neck at four different times; the flow direction is indicated by the arrow. The rectangles in the second image show the positions where the DNA extensions are measured. Main figure: Plot of $h_{\min }(t)$ (dotted line) and elongation rate $\dot{\varepsilon}(t)$ (blue line). Note that the elongation rate is constant in the filament regime and adopts a value $\dot{\varepsilon}_{0}$. Dashed line: Exponential fit of $h_{\min }(t)$. Inset: Normalized $h_{\min }(t)$ for the PAA solution (black line) and the solvent (open circles; $30 \%$ sucrose, $10-\mathrm{mM} \mathrm{NaCl}$ ), and total strain $\varepsilon(t)$ (green line; see text). 
the instant when the fluid neck between the drop and the capillary starts to thin, as illustrated in Fig. 1. The quantity $\varepsilon$ increases fast and reaches values near 4 at the transition. It then continues to increase, but more slowly, in the exponential regime to reach values near 7 at the end of this regime, indicating high deformation of fluid elements. At longer times, the exponential thinning regime stops as the filament starts to thin faster near the capillary or the drop (see Fig. 1). This result gives rise to a secondary filament that thins faster than the initial filament. Secondary filaments have been observed before [20].

To examine the dynamics at the molecular level, we monitored the extension of fluorescently labeled DNA molecules embedded in the polymer solution during the thinning process. While it would have been preferable to monitor the PAA molecules themselves, it is, at present, very difficult to stain large molecular weight synthetic polymers with fluorescent dyes. We have thus used stained DNA, of comparable length to the PAA molecules, as markers and probes of the properties of the polymers in solution [23-25], and we assume that their extension reflects that of the surrounding PAA molecules. Images of the stained DNA molecules are shown in Fig. 2. Before the onset of the exponential regime, the DNA probes are all coiled into small-diameter dots, as shown in Fig. 2(a) despite the relatively large shear rates present in the injection tube. At the small injection flux used here, the polymers have enough time to relax their extension as they are advected from the exit of the capillary to the neck region (see Ref. [26]). At the onset of the exponential regime, the molecules elongate suddenly, as shown in Figs. 2(b) and 2(c), signaling a transition towards elongated molecules in the direction of stretching. Recent measurements of molecular extensions during droplet pinch off also uncovered a transition to elongated conformations in the case of DNA solutions [27]; however, these measurements were not carried out in the elasto-capillary regime of interest here. The lengths of all the molecules that are in focus are then measured. Since the molecules move during the thinning of the filament, the measurement of the molecular extension can be difficult because of motion blur. This difficulty can be circumvented by using the properties of the velocity field within the filament. Indeed, the existence of a stagnation point in the flow (see below for velocity measurements) within the filament allows observations of molecular extensions in a region with little mean flow. In addition, and since the velocity can be measured for each DNA molecule analyzed, corrections for motion blur can be carried out (see Ref. [26]). The use of appropriate visualization tools (see the Appendix) and a precise knowledge of the velocity field within the filament also allowed us to set the correct experimental conditions (exposure time, correct LASER pulse width, and the correct frame rate) for proper visualizations of the DNA molecules (see the Appendix and Ref. [26]). The measured lengths $L$ are shown in Fig. 2(e), along with the thinning dynamics: Each dot represents a measurement on an isolated molecule within the filament. Note that the lengths measured are broadly distributed and span extensions from 0.5 to $25 \mu \mathrm{m}$. Probability density functions (pdf) of $L$ are then calculated before the transition $(t<0.125 \mathrm{~s})$ and after the transition $(t>0.125 \mathrm{~s})$, as shown in Fig. 2(f). The distribution is clearly wider after the transition, with the most probable length remaining near $1 \mu \mathrm{m}$. The pdfs are calculated for more than 700 measurements before and after the transition (which occurs at roughly $t=0.125 \mathrm{~s}$ ) in 70 drop breakup events. For each drop breakup event, the transition time is determined from inspecting the velocity of the molecules: Near the transition, the velocity of the molecules is very large and no measurements can be carried out. Right after the transition, the molecules slow down and length measurements can be carried out. This instant is located with a precision of about $0.03 \mathrm{~s}$ and is used to order the measurements in time.

To examine the temporal variation of the extensions during the visco-elasto-capillary regime, we separated the data into three groups and plotted the pdfs of $L$ for each group in Fig. 2(g): The pdfs are not modified during the exponential thinning phase, and they remain roughly the same, independently of the lifetime of the filament. The separation into three successive time intervals allows us to keep a sufficient number of measured lengths to calculate a pdf. The mean DNA length $\mathrm{L}_{m}$ averaged over intervals of $50 \mathrm{~ms}$ is plotted in the inset of Fig. 2(e). This length increases by a factor of 5 near the transition but remains constant during the exponential thinning regime, despite the fact that the total strain continues to increase. It is possible that since the Weissenberg number (defined as $\dot{\varepsilon}_{0} \lambda$ ) is close to 1 and just above the coil-stretch transition value of $1 / 2$ in the filament regime, as expected $[18,28]$, the molecular extension reaches a steady state due to partial relaxation of the molecules in the exponential regime. While a comparison with single-molecule experiments in elongational flows is difficult to carry out since our solutions are more concentrated, it is interesting to note that broad distributions of molecular extensions have been obtained before in elongational flows and that near the coilstretch transition of $1 / 2$ the evolution of mean extension with total strain is very slow [2]. Furthermore, and in order to stretch single-polymer molecules up to their full extension, the accumulated strain needs to be large and typically greater than 5 [29], especially for low Weissenberg numbers, as is the case here. While the maximum accumulated strain here does reach values near 7 at the end of the exponential regime (see Fig. 1), if measured from the beginning of the thinning of the neck including the Newtonian regime, it is possible that the filament experiences less accumulated strain if the Newtonian regime contributes less. The exact reasons for the stationarity of these distributions, though, call for additional work. 

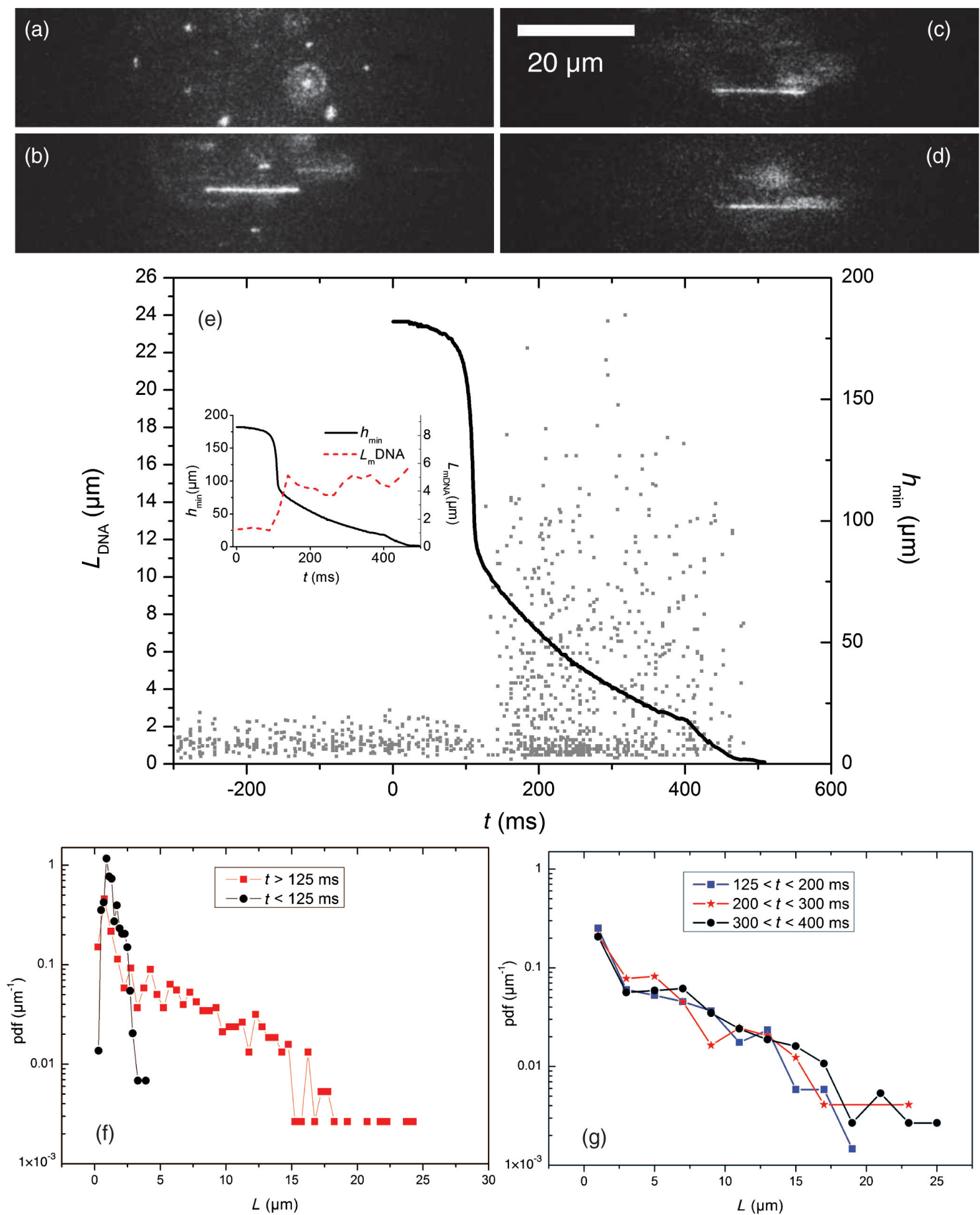

FIG. 2. Molecular extensions: (a) Coiled DNA molecules in the drop. (b) Coiled and stretched DNA molecules during the exponential thinning regime. (c) and (d) The same molecule at two different times separated by $130 \mathrm{~ms}$. (e) $h_{\text {min }}(t)$ and measured DNA lengths $L$ (squares). The data points for $L$ correspond to several runs from 70 different breakup events. Each run provides a few molecules for analysis at each instant of time. For each single drop event, we obtain a number of images at different times during the thinning. The data shown are the superposition of all such measurements during the thinning of the filament. Inset: $h_{\text {min }}(t)$ (line) and mean DNA length $L_{m}$ (dashed line). (f) Pdf $(L)$ before (black dots) and after (red squares) the onset of the exponential regime. (g) Pdf $(L)$ corresponding to three different time intervals during the exponential regime.

As mentioned earlier, this elasto-capillary regime [19] has been the subject of different studies and is often used to extract intrinsic properties of the solution, especially in the dilute limit and for low solvent viscosities, conditions for which other techniques are not suitable. In such filamentthinning experiments $[6,9,30]$, the elongational viscosity is deduced from the measurement of $h_{\min }$ using a force balance between the capillarity, which drives the thinning, 
and the elongational viscosity, which opposes it. This yields $\eta_{e} \dot{\varepsilon}_{0}=\frac{2 \gamma}{h_{\min }}$, giving an elongational viscosity $\eta_{e}$ that grows exponentially in time. An increase in elongational viscosity, and therefore in the elongational stress, is generally associated with an increase in the extension of the polymers [31]. Our experiments show that the mean length of the polymers remains unchanged, indicating that the elongational stress should remain constant as well, in contradiction with the above simple estimate. In a second method, using a filament stretching rheometer (FSR), which is well adapted for high-viscosity solutions, a liquid bridge is formed between two end plates that are separated from each other in a controlled fashion such that the elongation rate is fixed. Monitoring the force needed to stretch the filament yields a reliable measurement of the elongational viscosity, which increases versus total strain to reach a plateau at total strains larger than about 5 $[11,16]$. The two different methods generally yield different behaviors for the elongational viscosity [9].

To obtain $\eta_{e}$ from the thinning of the neck, we propose a more complete method that takes into account the full details of the neck dynamics and not just the balance of stresses in the filament. The Navier-Stokes equation in the one-dimensional approximation of the flow in the liquid bridge can be written as $\rho\left(\frac{\partial v}{\partial t}+v \frac{\partial v}{\partial z}\right)=-\gamma \frac{\partial \kappa}{\partial z}+3 \eta_{s} \frac{1}{h^{2}} \times$ $\frac{\partial}{\partial z}\left(h^{2} \frac{\partial v}{\partial z}\right)+\frac{1}{h^{2}} \frac{\partial}{\partial z}\left[h^{2}\left(\sigma_{z z}-\sigma_{r r}\right)\right][28,32]$. Here, $\rho$ is the
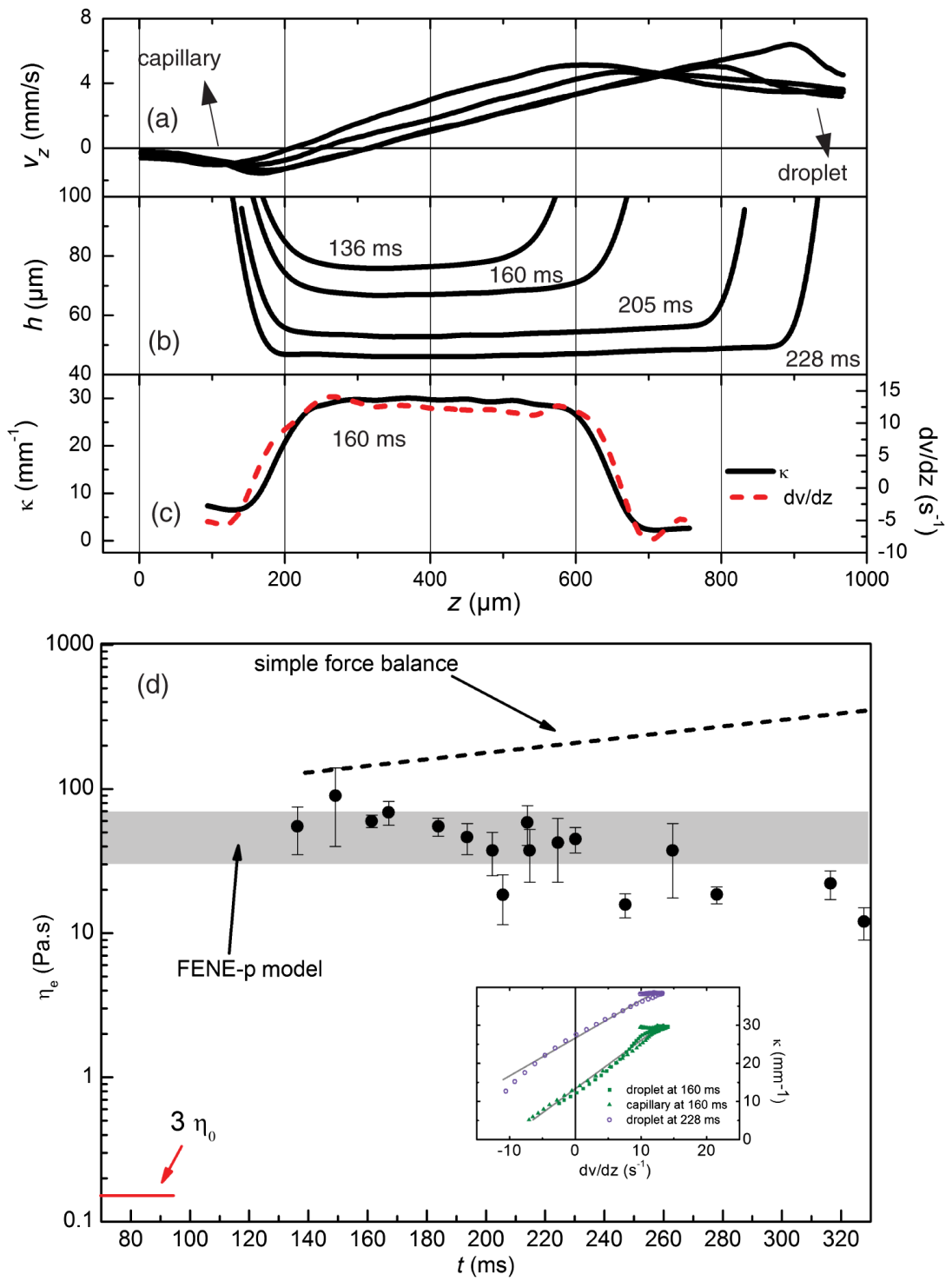

FIG. 3. Extensional viscosity: (a) Axial velocity profiles and (b) filament diameter at four different times during the exponential regime. (c) $\kappa$ (solid line) and $\dot{\varepsilon}=\frac{\partial v}{\partial z}$ (dashed line). (d) $\eta_{e}$ (dots) extracted from $\kappa$ versus $\dot{\varepsilon}$. Dashed line: $\eta_{e}$ using the force balance within the filament only. Note the low value of the extensional viscosity at early times deduced from the low shear viscosity of the solution and from the similarity in the early-time dynamics between the polymer solution and the reference Newtonian fluid. Gray region: Model values, taking into account the uncertainty on the value of the solution relaxation time. Inset: $\kappa$ versus $\dot{\varepsilon}$ for two different times during the exponential regime. 
density of the fluid, $\eta_{s}$ is the viscosity of the solvent, $\sigma_{z z}$ and $\sigma_{r r}$ are the diagonal terms of the stress tensor expressing the polymer contribution, and $\kappa=\frac{1}{r\left(1+r_{z}^{2}\right)^{1 / 2}}-\frac{r_{z z}}{\left(1+r_{z}^{2}\right)^{3 / 2}}$ is the curvature of the filament. Here, $r=h / 2$ is the radius, and $\mathrm{r}_{z}$ and $\mathrm{r}_{z z}$ are the first- and second-order derivatives of $r$ with respect to $z$. The solvent contribution is considered to be negligible. Since $\eta_{e}$ is defined by $\eta_{e} \dot{\varepsilon}=\sigma_{z z}-\sigma_{r r}$, the last term of the equation can be written as $\eta_{e}\left(\frac{\partial^{2} v}{\partial z^{2}}+\frac{2}{h} \frac{\partial v}{\partial z} \frac{\partial h}{\partial z}\right)$. This term can be approximated by $\eta_{e} \frac{\partial^{2} v}{\partial z^{2}}$, leading to $\kappa=\frac{\eta_{e}}{\gamma} \frac{\partial v}{\partial z}+C$. The constant $C$ is independent of $z$ but may depend on time. While this relation is consistent with $\eta_{e}=\frac{2 \gamma}{h \dot{\varepsilon}_{0}}$, as in the filament thinning method, it has the advantage of providing the full variation of the stress-versus-strain rate. In this analysis, we have neglected the effects of outer flow for two reasons: The first reason is that an estimate of the effect of this outer flow using a method proposed in [12] turns out to be negligible, and second, varying the outer flux has no noticeable effect on the thinning dynamics in the elasto-capillary regime. (See Ref. [26], where thinning dynamics for different outer-flow fluxes is shown: No noticeable change with flux is observed. It is possible that the use of a coflow system instead of a flow-focusing system is responsible for the observed independence of the thinning dynamics versus outer-fluid flux, at least for the flux range used here.) We compute the elongation rate $\frac{\partial v}{\partial z}=\dot{\varepsilon}$ from the velocity measurements in Fig. 3(a) and the curvature $\kappa$ from the shape of the filament in Fig. 3(b). The variation of $v(z)$ versus $z$ is linear in the filament region, with a well-defined stagnation point, giving an extension rate that is equal to $\dot{\varepsilon}_{0}$. The velocity then matches to that in the drop or in the capillary by going through a local maximum or minimum. These features are in qualitative agreement with recent measurements [33]. The profile of the neck, shown in Fig. 3(b), shows a well-defined filament with a constant diameter. The local diameter then increases near the drop and the capillary. The curvature and elongation rate, shown in Fig. 3(c), both show a plateau in the central region of the filament and decrease as the capillary or the drop is approached. The variation of $\kappa$ versus $\frac{\partial v}{\partial z}$, shown in the inset of Fig. 3(d), is roughly linear, with a slope proportional to $\eta_{e}$. The balance invoked above, $\eta_{e} \dot{\varepsilon}_{0}=\frac{2 \gamma}{h_{\min }}$, represents a single point on this graph at $\dot{\varepsilon}=\dot{\varepsilon}_{0}$, while the full relation gives the full evolution of the stress-versus-strain rate. The elongational viscosity, extracted from the slope of the variation of $\kappa$ versus $\dot{\varepsilon}$, for different times, Fig. 3(d), is of the same order of magnitude as that obtained from the simple balance stated above, at least for early times. However, the temporal variation is very different; instead of an increase versus time, $\eta_{e}$ seems to be roughly constant within the accuracy of our measurements. The initial times may have a slightly higher $\eta_{e}$ than the later times, but our accuracy is not sufficient to confirm this trend. A roughly constant $\eta_{e}$ is consistent with the fact that the extension of the polymers is constant in time. An estimate of $\eta_{e}$ using the FENE-P model [34] yields a value of $50 \pm 20 \mathrm{~Pa} \mathrm{~s}$, in agreement with our estimates (see the Appendix). Additional experiments using different polymer concentrations also show a reasonable agreement with the model (see Ref. [26]). Furthermore, and for total strains higher than about 5, previous FSR experiments $[11,16,35]$ show that $\eta_{e}$ reaches a plateau value. In the exponential regime, the maximum total strain here is between 4 and 7 , so a constant $\eta_{e}$ is consistent with the general trend obtained in FSR experiments even though no results are available for such low-viscosity solutions using this method [11]. To compare our results with such experiments, we focus on the Trouton ratio (i.e., $\eta_{e} / \eta$, where $\eta$ is the low shear viscosity of the solution); for solutions with molecular weight and concentration comparable to ours, the values obtained are of order 1000 for large strains, which is the case in our experiments $[11,36]$. Further, some experiments using the filament-thinning method uncovered a nearly linear regime before the breakup of the filaments $[15,30]$; the elongational viscosity extracted for conditions nearly similar to our solution using this regime gives values near $100 \mathrm{~Pa}$ s, not far from our estimate.

\section{DISCUSSION}

The rupture of fluid necks provides a unique situation to study the interaction between the constituents of a complex fluid and the flow field. For polymer solutions, the polymer molecules remain in a coiled state and elongate suddenly when the deformation of the neck is sufficiently strong. The extension of the polymers in the neck region enhances the elongational viscosity of the solution by over 2 orders of magnitude, leading to an inhibition of the rupture dynamics and the formation of long cylindrical filaments. During the thinning of this filament, the distribution of polymer extensions remains roughly the same, indicating the onset of a stationary state. The elongational viscosity extracted from an analysis of the full dynamics of the neck reveals that this quantity is very high compared to the shear viscosity of the solution, but it remains roughly the same as the lifetime of the filament increases in accordance with the distribution of polymer extensions. Besides its importance for understanding the link between microscopic and macroscopic properties of complex fluids, slender filaments of the sort used here may be useful for stretching macromolecules for different purposes, such as inducing anisotropy of polymeric materials by simply depositing such filaments on different substrates or for optical screening purposes where elongated biomolecules need to be stretched to be presented for analysis. For this latter application, fluid necks have the advantage of avoiding the presence of walls that may hinder the screening. The periodic nature of the droplet pinch off may also allow for a high throughput of molecules for analysis. While here we have used the detachment of droplets to produce the 
filaments, a liquid bridge between two capillaries embedded in a microfluidic channel can also be used to produce these filaments. This latter situation would have the advantage of suppressing the mean flow due to the droplet motion and would allow for sharper images of the macromolecules for screening purposes.

\section{APPENDIX: MATERIALS AND METHODS}

The experiments use a microfluidic channel made of glass capillaries in a coflow configuration. Droplets are detached periodically from a small round glass capillary embedded in a larger rectangular one (see Fig. 1). Using two syringe pumps, the polymer solution is injected into the round capillary at a flow rate of $Q_{a q}=50 \mu \mathrm{l} / \mathrm{hr}$, while the continuous phase (hexadecane) is injected into the rectangular capillary at $Q_{\mathrm{oil}}=20 \mathrm{ml} / \mathrm{hr}$. The capillaries are rendered hydrophobic using OTS [Trichloro(octadecyl) silane] to avoid the use of surfactants, as they change the dynamics of the breakup process $[37,38]$. The formation of droplets and their detachment is monitored with an inverted microscope, with $\mathrm{X} 10$ or X20 objectives in a bright-field configuration, and a high-speed camera working at 2000 frames per second (fps). The polymer used is polyacrylamide (PAA, $M_{w}=18.10^{6} \mathrm{~g} / \mathrm{mol}$ ) at a concentration of $300 \mathrm{ppm}$ by weight in a buffer solution with $10-\mathrm{mM} \mathrm{NaCl}$ and $30 \%$ sucrose; the viscosity of the solvent is $\eta_{s}=3 \mathrm{mPa}$. The low shear viscosity of our solutions is $45 \mathrm{mPa}$ s. We monitor the flow field within the filament by particle tracking velocimetry (PTV), with solutions seeded with $1-\mu \mathrm{m}$ polystyrene spheres. To monitor the polymer conformations during the thinning, the polymer solution was seeded with flexible polymers T4 DNA [fluorescently labeled with POPO-3 (molecular probes) at a 1:5 dye-base ratio] [1]. The radius of gyration and the Contour length $\left(L_{c}\right)$ of the two polymers are $1.3 \mu \mathrm{m}$ and $56 \mu \mathrm{m}$ for T4 DNA and $0.33 \mu \mathrm{m}$ and $53 \mu \mathrm{m}$ for the PAA [39]. Despite the slight difference in length, we expect these two polymers to behave roughly similarly with respect to the flow field. The concentration of DNA in the solution used is $8 \mathrm{ppm}$; the addition of DNA to the PAA solution does not change the thinning dynamics of the filament. The DNA molecules are observed in an epifluorescence configuration with a X100 (oil immersion, N.A. $=1.25$ ) objective with a working distance of $290 \mu \mathrm{m}$ to monitor the DNA molecules within the filament. The dyes are excited with a continuous laser $(532 \mathrm{~nm}, 425 \mathrm{~mW})$ triggered with a function generator. The exposure time of $3 \mathrm{~ms}$ is fixed by the pulse width. A sensitive camera (EM CCD) working at $100 \mathrm{fps}$ is used. The total field of view for the fluorescence setup is $100 \mu \mathrm{m}$ and is located near the stagnation point in the filament so as to minimize motion blur. The rheology of the polymer solution was characterized using a $60-\mathrm{mm}$, $0.5^{\circ}$-cone-plate geometry in an AR rheometer. The characteristic time of the polymer solution was estimated to be $\lambda=0.1 \pm 0.025 \mathrm{~s}$ from rheology measurements.
The surface tension of the interface PAA solution/ hexadecane is $\gamma=47 \pm 2 \mathrm{mN} / \mathrm{m}$.

The extensional viscosity from the FENE-P model is estimated as $\eta_{e}^{\infty}=3 \eta_{s}+2 \frac{c}{M} R T \lambda b\left(1-\frac{1}{2 \lambda \epsilon_{0}}\right)$. Here, $c$ is the concentration in ppm, $M$ is the molecular weight of the polymer, $R$ is the gas constant, $T$ is the temperature, and $b$ is the so-called finite extensibility parameter whose value was taken to be 10000 , as obtained from normal stress measurements [12].

[1] T. T. Perkins, S.R. Quake, D.E. Smith, and S. Chu, Relaxation of a Single DNA Molecule Observed by Optical Microscopy, Science 264, 822 (1994).

[2] T. T. Perkins, D. E. Smith, and S. Chu, Single Polymer Dynamics in an Elongational Flow, Science 276, 2016 (1997).

[3] C. M. Schroeder, H. P. Babcock, E.S. G. Shaqfeh, and S. Chu, Observation of Polymer Conformation Hysteresis in Extensional Flow, Science 301, 1515 (2003).

[4] R. G. Larson, The Rheology of Dilute Solutions of Flexible Polymers: Progress and Problems, J. Rheol. 49, 1 (2005).

[5] A. Groisman and V. Steinberg, Elastic Turbulence in a Polymer Solution Flow, Nature (London) 405, 53 (2000).

[6] Y. Amarouchene, D. Bonn, J. Meunier, and H. Kellay, Inhibition of the Finite-Time Singularity during Droplet Fission of a Polymeric Fluid, Phys. Rev. Lett. 86, 3558 (2001).

[7] A. Gyr and H.W. Bewerdsdorff, Drag Reduction of Turbulent Flows by Additives (Kluwer, Dordrecht, 1995).

[8] J. Eggers, Nonlinear Dynamics and Breakup of FreeSurface Flows, Rev. Mod. Phys. 69, 865 (1997).

[9] S. L. Anna and G. McKinley, Elasto-Capillary Thinning and Breakup of Model Elastic Liquids, J. Rheol. 45, 115 (2001).

[10] R. Sattler, A. Kityk, and C. Wagner, Molecular Configurations in the Droplet Detachment Process of a Complex Liquid, Phys. Rev. E 75, 1 (2007).

[11] G.H. McKinley and T. Sridhar, Filament-Stretching Rheometry of Complex Fluids, Annu. Rev. Fluid Mech. 34, 375 (2002).

[12] P.E. Arratia, J. P. Gollub, and D. J. Durian, Polymeric Filament Thinning and Breakup in Microchannels, Phys. Rev. E 77, 1 (2008).

[13] M. Roché, H. Kellay, and H. A. Stone, Heterogeneity and the Role of Normal Stresses during the Extensional Thinning of Non-Brownian Shear-Thickening Fluids, Phys. Rev. Lett. 107, 13 (2011).

[14] J.P. Rothstein, Transient Extensional Rheology of Wormlike Micelle Solutions, J. Rheol. 47, 1227 (2003).

[15] M. Stelter, G. Brenn, A. L. Yarin, R. P. Singh, and F. Durst, Investigation of the Elongational Behavior of Polymer Solutions by Means of an Elongational Rheometer, J. Rheol. 46, 507 (2002).

[16] V. T. Tirtaatmadja and T. Sridhar, A Filament Stretching Device for Measurement of Extensional Viscosity, J. Rheol. 37, 1081 (1993).

[17] A. V. Bazilevsky, V. M. Entov, and A. N. Rozhkov, Liquid Filament Microrheometer and Some of its Applications, 
Proceedings of the 3rd European Rheology Conference, edited by D. R. Oliver (Elsevier, London and New York, 1990), p. 41.

[18] V. M. Entov and E.J. Hinch, Effect of a Spectrum of Relaxation Times on the Capillary Thinning of a Filament of Elastic Liquid, J. Non-Newtonian Fluid Mech. 72, 31 (1997).

[19] G.H. McKinley, Visco-Elasto-Capillary Thinning and Break-Up of Complex Fluids, in Annual Rheology Reviews, edited by D. M. Binding and K. Walters (British Society for Rheology, Aberystwyth, 2005), pp. 1-48.

[20] R. Sattler, C. Wagner, and J. Eggers, Blistering Pattern and Formation of Nanofibers in Capillary Thinning of Polymer Solutions, Phys. Rev. Lett. 100, 3 (2008).

[21] J. Husny and J. J. Cooper-White, The Effect of Elasticity on Drop Creation in T-Shaped Microchannels, J. NonNewtonian Fluid Mech. 137, 121 (2006).

[22] B. Steinhaus, A. Q. Shen, and R. Sureshkumar, Dynamics of Viscoelastic Fluid Filaments in Microfluidic Devices, Phys. Fluids 19, 073103 (2007).

[23] N. François, D. Lasne, Y. Amarouchene, B. Lounis, and H. Kellay, Drag Enhancement with Polymers, Phys. Rev. Lett. 100, 1 (2008).

[24] N. François, Y. Amarouchene, B. Lounis, and H. Kellay, Polymer Conformations and Hysteretic Stresses in Nonstationary Flows of Polymer Solutions, Europhys. Lett. 86, 34002 (2009).

[25] M. I. Smith and V. Bertola, Effect of Polymer Additives on the Wetting of Impacting Droplets, Phys. Rev. Lett. 104, 154502 (2010).

[26] See Supplemental Material at http://link.aps.org/ supplemental/10.1103/PhysRevX.3.041002 for additional data showing the effects of concentration, effects of flux, and image analysis.

[27] G. Juarez and P. E. Arratia, Extensional Rheology of DNA Suspensions in Microfluidic Devices, Soft Matter 7, 9444 (2011).

[28] C. Clasen, J. Eggers, M. A. Fontelos, J. Li, and G. H. Mckinley, The Beads-on-String Structure of Viscoelastic Threads, J. Fluid Mech. 556, 283 (2006).
[29] T. T. Perkins, Ph.D. thesis, Stanford University, 1997.

[30] M. Stelter, G. Brenn, A. L. Yarin, R. P. Singh, and F. Durst, Validation and Application of a Novel Elongational Device for Polymer Solutions, J. Rheol. 44, 595 (2000).

[31] R. G. Larson, The Structure and Rheology of Complex Fluids (Oxford University Press, New York, 1990).

[32] M. Forest and Q. Wang, Change of Type Behavior in Viscoelastic Slender Jet Models, Theor. Comput. Fluid Dyn. 2, 1 (1990).

[33] S. Gier and C. Wagner, Visualization of the Flow Profile Inside a Thinning Filament During Capillary Breakup of a Polymer Solution via Particle Image Velocimetry and Particle Tracking Velocimetry, Phys. Fluids 24, 053102 (2012).

[34] R. B. Bird, C.F. Curtiss, R. C. Armstrong, and O. Hassager, Dynamics of Polymeric Liquids (John Wiley \& Sons, New York, 1987), Vol. 2.

[35] S. L. Anna, G. H. McKinley, D. A. Nguyen, T. Sridhar, S. J. Muller, J. Huang, and D. F. James, An Interlaboratory Comparison of Measurements from Filament-Stretching Rheometers Using Common Test Fluids, J. Rheol. 45, 83 (2001).

[36] R. K. Gupta, D. A. Nguyen, and T. Sridhar, Extensional Viscosity of Dilute Polystyrene Solutions: Effect of Concentration and Molecular Weight, Phys. Fluids 12, 1296 (2000).

[37] M. Roché, M. Aytouna, D. Bonn, and H. Kellay, Effect of Surface Tension Variations on the Pinch-Off Behavior of Small Fluid Drops in the Presence of Surfactants, Phys. Rev. Lett. 103, 264501 (2009).

[38] M. Robert De Saint Vincent, J. Petit, M. Aytouna, J. P. Delville, D. Bonn, and H. Kellay, Dynamic Interfacial Tension Effects in the Rupture of Liquid Necks, J. Fluid Mech. 692, 499 (2012).

[39] Y. Liu, Y. Jun, and V. Steinberg, Concentration Dependence of the Longest Relaxation Times of Dilute and Semi-Dilute Polymer Solutions, J. Rheol. 53, 1069 (2009). 